FPH OH teams are required to provide direction and support in addressing community health issues and corporate health social responsibility programs, expanding scope to include occupational, environmental, and public health. This paper describes how this was achieved.

Methods To institutionalise $\mathrm{OH}$ in the Environment, Safety, and Health Management System (ESH MS) the following were implemented:

a. Developed a set of mandatory $\mathrm{OH}$ standards including requiring companies to strategically address environmental

health and CSR health programs;

b. Established $\mathrm{OH}$ Management System;

c. Implemented health guidelines and procedures; and

d. Implemented $\mathrm{OH}$ competency development.

Result The FPH Corporate Health function was established providing technical support, guidance, and services in the development, implementation, monitoring, and evaluation of all health initiatives including a 'one-stop-shop' for all health needs.

Discussion Philippine $\mathrm{OH}$ focuses on medical services, reactive in approach, and programs are not risk based. Corporate culture and perspective needs to change to take on a proactive and risk based approach. Health programs needs to be simplified, standardised, and made fit-for-purpose. The $\mathrm{OH}$ management system of FPH has addressed this through organisational development, engagement of stakeholders, and managing health just like any other business.

\section{DEVELOPING OCCUPATIONAL HEALTH BEYOND COMPLIANCE}

Rishikesh Naik*. VP, Occupational Health and Hygiene, Aditya Birla Group, Mumbai, India

\subsection{6/oemed-2018-ICOHabstracts.893}

Introduction Monitoring and development of occupational health in a large multinational conglomerate is always a demanding task in view of challenges like diverse nature of manufacturing processes, multiple geographies and its legal structure, diverse social and cultural background, different level of awareness etc. Aditya Birla group is 41 billion USD conglomerate having footprint in 12 different businesses across more than 34 countries. Driving occupational health with standardisation of practices across such a large organisation warrants unique approach and intervention.

Methods At Aditya Birla Group, Occupational health has been adopted as fundamental element of overall inclusive approach for sustainable business practices. The framework is created incorporating international standards beyond local legal requirements applicable to all businesses/geographies and self-assessment process has been adopted to identify current status and gaps. This has in turn resulted in roadmap to bridge these gaps.

Result Over 90 different sites of the group have completed self-assessment questionnaire (SAQs), current status and gaps were identified to work up on. Awareness on required aspects of occupational health has been provided based on gaps identified and best practices within the groups were shared. This has resulted into overall improvement of occupational health status across the group which is measurable in terms of selfassessment score.

Discussion Management of occupational health across geographies at multiple sites is always a challenge. A focused and unique approach of self-assessment questionnaire (SAQ) along with gap identification has been found very useful to monitor and support development of occupational health status across large organisation while keeping ownership with local management.

\section{OCULAR MORBIDITY AMONG WELDERS IN THE SHIPBUILDING INDUSTRY, GOA}

${ }^{1}$ GV Prabhu* ${ }^{2}$ Nateshan Bhumika, ${ }^{3}$ Agnelo Menino Ferreira, ${ }^{4}$ Zile Singh. ${ }^{1}$ Advisor (Medical and OHSE), Goa Shipyard Limited, Vasco da gama, Goa, India; ${ }^{2}$ Assistant Professor, Department of Community Medicine, Pondicherny Institute of Medical Sciences, India; ${ }^{3}$ Director Professor, Preventive and Social Medicine, Goa Medical College, Goa, India; ${ }^{4}$ Professor and Head, Department of Community Medicine, Pondicherry Institute of Medical Sciences, India

\subsection{6/oemed-2018-ICOHabstracts.894}

Introduction It is known that the shipbuilding industry relies heavily on welding. While welding has conventionally been known to predispose welders to ocular morbidity (OM), organised sector requires implementation of stringent workplace safety rules. Are welders still predisposed to OM by virtue of their occupation?

Methods This cross-sectional study involved 552 workers; 276 welders and 276 nonwelders. An interviewer-administered questionnaire was followed by ocular examination and testing by means of a Titmus Vision Tester.

Result The prevalence of OM among the two study groups comparable in age and duration of employment (DOE), was found to be significantly higher among welders with odds ratio (OR) of $1.75 \quad(95 \%$ confidence interval CI: 1.45 to 2.11)) despite regular PPE (personal protective equipment) use. Arc eye was limited to welders. Prevalence of cataract was greater in welders $(\mathrm{OR}=3.60,95 \% \mathrm{CI}: 2.27$ to 5.70$)$ and was associated with a younger age and a shorter DOE compared to nonwelders. There were more cases of diminished colour vision among the welders $(\mathrm{OR}=4.09,95 \% \mathrm{CI}: 1.63$ to 10.28) and they did not differ significantly from the nonwelders with the same ocular morbidity in terms of mean age and DOE. Visual field defects, pterygium and myopia were more prevalent among welders; however statistical significance was weak

Discussion Welders have a greater burden of ocular morbidity compared to nonwelders despite regular PPE use. Further investigation to identify underline issues to enable amendment of workplace regulation, screening criteria and worker education material and propose access to evidence based recommendation tailor-made to the actual workplace situation.

\section{ERGONOMIC ASSESSMENT OF ARC WELDING JOB IN CONSTRUCTION INDUSTRY}

'Somnath Gangopadhyay*, 'Shreya Sinha. 'Professor Occupational Ergonomics Laboratory Department of Physiology University of Calcutta Calcutta, India; ${ }^{2}$ Research Fellow Occupational Ergonomics Laboratory Department of Physiology University of Calcutta Calcutta, India

\subsection{6/oemed-2018-ICOHabstracts.895}

Introduction Among the various process of welding metal arc welding is the most common, versatile and inexpensive one and account for over 50\% of the total welding in advanced countries and over $80 \%$ in India. A large number of workforces around the world earn their livelihood in this occupation. Welders have no fixed work station in developing world 